\title{
MERGELYAN TYPE THEOREMS FOR SOME FUNCTION SPACES
}

\author{
Arne Stray
}

\begin{abstract}
Let $F$ be a relatively closed subset of the unit $\operatorname{disc} D$. If $A$ is any of the Hardy spaces $H^{p}(D), 0<p<\infty, \overline{\left.A\right|_{F}}$ denotes the functions on $F$ being uniform limits of elements from $H^{p}(D)$. Let $\tilde{F}$ consist of all $z \in D$ such that $|f(z)| \leq \sup \{|f(z)| z \in F\}$ for any bounded analytic function in $D$. It is proved that $\overline{\left.A\right|_{F}}$ consist of all functions $f$ that can be decomposed as $f=u+v$, where $u$ belongs to $H^{p}(D)$ and $v$ is a uniformly continuous function on the set $\tilde{F}$, analytic at interior points of $\tilde{F}$.
\end{abstract}

Let $A$ be a linear space of analytic functions and $F$ a subset of the complex plane $\mathcal{C}$ such that each $f \in A$ is defined on $F$. We denote by $\overline{\left.A\right|_{F}}$ the functions being uniformly approximable on $F$ by sequences from $A$. The aim with this paper is to give a partial solution to problem 8.5 no. 2 in [7]. If $A$ is any of the classical Hardy spaces $H^{p}(D), 0<p<\infty$, our main result is that $\overline{\left.A\right|_{F}}$ coincides (modulo the approximating space) with a well defined algebra of uniformly continuous analytic functions on $F$.

Before giving a precise formulation of the main result, we need some definitions.

Let $C_{u a}(F)$ denote the functions on $F$ being analytic in its interior $F^{0}$ and admitting continuous extension to the extended complex plane $\mathcal{C} \cup\{\infty\}$. If $F$ is a compact subset of $\mathcal{C}$ and $P$ consists of the polynomials, a famous theorem of S. N. Mergelyan [7] can be formulated as

$$
\overline{\left.P\right|_{F}}=C_{u a}(\tilde{F})
$$

where $\tilde{F}$ is the union of $F$ and the bounded components of $C / F$. 
Suppose now we replace $P$ by the set $H(\mathcal{C})$ consisting of all entire functions. Also allow $F$ to be a closed but possibly unbounded subset of $\mathcal{C}$. Then it can be proved that

$$
\overline{\left.H(\mathcal{C})\right|_{F}}=H(\mathcal{C})+C_{u a}(\tilde{F})
$$

where $\tilde{F}$ is the union of $F$ and certain components of $C / F$. A component $V$ is to be included in $\tilde{F}$ if and only if $V \cup\{\infty\}$ is not arcwise connected in $C \cup\{\infty\}$. For details see [8], [9] and [10].

In general $A$ may contain unbounded functions. For this reason it is natural to look for an identity like (I) if we seek to describe $\overline{A \mid F}$ in terms of uniformly continuous analytic functions. Let us use the notation $\|g\|_{B}=\sup \{|g(x)|: z \in B\}$ if $g$ is a function defined on the set $B$. We also define the hull of $F$ with respect to $A$ :

$$
\hat{F}^{A}=\left\{z:|f(z)| \leq\|f\|_{F}, \quad f \in A\right\} .
$$

We look for spaces $A$ satisfying the following:

$$
\overline{A_{\mid F}}=A+C_{u a}\left(\hat{F}^{A}\right) .
$$

Our main result is the $(*)$ is valid for the classical Hardy sapces $H^{P}(D)$ in the unit disc $D, 0<p<\infty$, when $F$ is any relatively closed subset of $D$. Also note that the two introductory examples are special cases of (*).

We refer to [3] or [5] for the basic theory of $H^{p}(D), 0<p \leq \infty$. In particular $H^{\infty}(D)$ denotes the bounded analytic functions in $D$. If $F \subset D$ is relatively closed, let

$$
\hat{F}=\left\{z \in D:|f(z)| \leq\|f\|_{F}, \quad f \in H^{\infty}(D)\right\} .
$$

Our main result is

Theorem 1. If $0<p<\infty$, then $\overline{\left.H^{p}(D)\right|_{F}}=H^{p}(D)+C_{u a}(\hat{F})$.

Proof of Theorem 1: If $f \in H^{p}(D)$ it is easy to find $\left\{f_{n}\right\} \subset H^{\infty}(D)$ such that $\left|f_{n}(z)\right| \leq|f(z)|$ and $f_{n}(z) \rightarrow f(z)$ for $z \in D$. This shows that $\hat{F}^{A}=\hat{F}$ if $A=H^{p}(D), 0<p<\infty$.

Let us first prove that $\overline{\left.H^{p}(D)\right|_{F}} \subset H^{p}(D)+C_{u a}(\hat{F})$. If $g \in \overline{\left.H^{p}(D)\right|_{F}}$ is bounded, we may assume

$$
g=\sum_{n} f_{n}, \quad f_{n} \in H^{p}(D)
$$


in the sense that $\sum_{n}\left\|f_{n}\right\|_{F}<\infty$.

There are two special classes of sets $F$ where a short proof of the decomposition of $g$ can be found. It may be instructive to consider these cases prior to the general proof.

Let us firs assume that $F$ is a Farrell set for $H^{p}(D)$. (See $[\mathbf{8}]$ for definition and various properties of these sets). Then we can find polynomials $p_{n}, n=1,2$ such that

$$
\left\|p_{n}\right\|_{F} \leq\left\|f_{n}\right\|_{F}+2^{-n}
$$

and

$$
\left\|p_{n}-f_{n}\right\|_{H^{p}} \leq 2^{-n}
$$

for $n=1,2, \ldots$. This gives a decomposition

$$
g=\sum_{n}\left(f_{n}-p_{n}\right)+\left.\sum_{n} p_{n}\right|_{\hat{F}}
$$

as claimed.

In our second example, we assume that $F$ can be written as a Blaschke sequence $S=\left\{\zeta_{\nu}\right\}$, meaning that

$$
\sum_{\nu} 1-\left|\zeta_{\nu}\right|<\infty
$$

Then it is well known that the Blashcke product

$$
B(z)=\Pi \frac{\left|\zeta_{\nu}\right|}{\zeta_{\nu}} \frac{\zeta_{\nu}-z}{1-\bar{\zeta}_{\nu} z}
$$

converge in $D$. Using cofinite subproducts $B_{n}$ of $B$, we can obtain

$$
\left\|\left(1-B_{n}\right) f_{n}\right\|_{H^{p}(D)}<2^{-n}, \quad n=1,2, \ldots
$$

and again we have a decomposition

$$
g=\sum_{n}\left(1-B_{n}\right) f_{n}+\left.\sum_{n} B_{n} f_{n}\right|_{F} .
$$

The geometric properties of the set $F$ are quite different in the two cases just discussed. To clarify this, let $\bar{F}_{n t}$ denote the non tangential closure of $F$ on the unit circle $T$. So $z \in \bar{F}_{n t}$ if $z \in T$ and $z$ is a limit of a sequence $\left\{z_{n}\right\} \subset F$ satisfying $\left|z-z_{n}\right| \leq C\left(1-\left|z_{n}\right|\right), n=1,2, \ldots$, where $C$ may depend only on $z$. We also define $\bar{F}_{t}=\bar{F} \cap \bar{T} \backslash \overline{F_{n t}}$. 
It is well known that $F$ is a Farrell set for $H^{p}(D)$ if and only if the linear measure $\left|\bar{F}_{t}\right|$ of $\bar{F}_{t}$ is zero $([\mathbf{8}])$. On the other hand, the condition (1) is easily seen to imply that $\left|\bar{F}_{n t}\right|=0$.

We have thus obtained the decomposition of $\overline{\left.H^{p}(D)\right|_{F}}$ in two rather different situations. The general proof will be divided into parts reflecting the "geometry" of the cases considered above. We shall argue as in the proof where $F$ was a Farrell set, but the polynomials $p_{n}$ will be replaced by functions from $H^{p}(D)$ having a uniformly continuous restriction to $F$.

The key part of the proof is an approximation argument related to the set $\bar{F}_{n t}$ :

Lemma 1. Given $f \in H^{p}(D), 0<p<\infty$, and $\epsilon>0$, there is an open set $V$ and $f_{1} \in H^{p}(D)$ with the following properties:

(i) $\left\|f-f_{1}\right\|_{H^{p}(D)}<\epsilon$

(ii) $\left\|f_{1}\right\|_{F}<\|f\|_{F}+\epsilon$

(iii) $f_{1}$ extends continuously to $\bar{F} \cap V$

(iv) $\left|\bar{F}_{n t} \backslash V\right|=0$.

For the moment we take Lemma 1 for granted. Consider the "tangential" part $\bar{F}_{t}$ of $\bar{F} \cap T$. Let $K$ be a compact subset of $\bar{F}_{t}$. We assume there is a number $\delta=\delta(K)$ such that $I_{z} \cap K=\phi$ if $z \in F,|z|>1-\delta$, and $I_{z}$ denotes the arc $I_{z}=\left\{e^{i \theta}:\left|z-e^{i \theta}\right| \leq 2(|-| z \mid)\right\}$.

By a construction due to J. Detraz ([2, Prop. 3.1]), we can find an outer function $G_{K} \in H^{\infty}(D)$ such that

$\left|G_{K}\right| \leq 1$ and $G_{K}(z) \rightarrow 0$ if $z \rightarrow K$ and $z \in F$. Moreover, $G_{K}$ extends to be continuous and non zero at any $e^{i \theta} \in T \backslash K$.

We can find an increasing sequence of such sets $K_{n} \subset \bar{F}_{t} \backslash V$ with corresponding outer functions $G_{n}$, such that $\left|\bar{F}_{t} \backslash V \backslash K_{n}\right| \rightarrow 0$ and such that $G=\prod_{n} G_{n}$ has the following properties

(i) $0<|G(z)| \leq 1, z \in D$

(ii) $G$ extends to be continuous at any $e^{i \theta} \in V \cap T$.

It also follows from the construction of $\left\{G_{n}\right\}$ that

$$
G(z) \rightarrow 0 \text { if } z \in F \text { and } z \rightarrow z_{0} \in \cup_{n} K_{n} .
$$

Consider finally the set

$$
L=(\bar{F} \bigcap T) \backslash V \backslash \bigcup_{n} K_{n}
$$


It is evident that the linear meausre $|L|$ of $L$ is zero. By a general version of the Rudin-Carleson theorem ([2]) there is $H \in H^{\infty}(D)$ with continuous extension to $L \cup(T \backslash \bar{L})$ such that $H \neq 0$ in $D$ and $H=0$ on $L$.

For $n=1,2, \ldots$ we consider the functions $U_{n}$ in $H^{p}(D)$ given by

$$
U_{n}=G^{\frac{1}{n}} H^{\frac{1}{n}} f_{1}
$$

where $f_{1}$ satisfies the conclusions of Lemma 1 .

It follows from the construction of $f_{1}, G$ and $H$, that $\left.U_{n}\right|_{F}$ is uniformly continuous. This implies that $\left.U_{n}\right|_{\hat{F}} \in C_{u a}(\hat{F})$, by the maximum principle. To be a little bit more specific, suppose $z_{0} \in \bar{F} \cap T$ and that $U_{n}(z) \rightarrow 0$ as $z \rightarrow z_{0}$ and $z \in F$. Then $\left|U_{n}\right|<\epsilon$ in $F \cap \Delta\left(z_{0}\right)$, for some disc centered at $z_{0}$. Choose a polynomial $p$ peaking at $z_{0}$ such that $\left|U_{n} p\right|<\epsilon$ on $F$. Then if $p\left(z_{0}\right)=1$, we have

$$
\limsup _{\substack{z \rightarrow z_{0} \\ z \in \hat{F}}}\left|U_{n}(z)\right|=\limsup _{\substack{z \rightarrow z_{0} \\ z \in \hat{F}}}\left|U_{n}(z) p(z)\right| \leq \epsilon
$$

since $\left\|U_{n} p\right\|_{\hat{F}} \leq\left\|U_{n} p\right\|_{\bar{F}} \leq \epsilon$.

We turn to the proof of Theorem 1. If $f \in H^{p}(D)$ and $\epsilon>0$ is given, we have shown (modulo proving Lemma 1 ) that there is a function $U=U_{n} \in H^{p}(D) \cap C_{u a}(\hat{F})$ with $n$ so large that

$$
\begin{array}{r}
\|f-U\|_{H^{p}(D)}<\epsilon \\
\|U\| \leq\|f\|_{F}+\epsilon .
\end{array}
$$

The proof of Theorem 1 now follows the introductory argument we gave in the special case where $F$ is a Farrell set.

Let us finally prove Lemma 1 . We may assume that $f$ is bounded in $D$.

So given $f \in H^{\infty}(D)$, and $\epsilon>0$, we consider a compact set $K \subset \bar{F}_{n t}$. We shall require several properties of $K$ related to $f$. If $0<\alpha<\pi$, $T(\theta, \alpha)$ denotes the cone in $D$ with opening angle $\alpha$, terminating at $e^{i \theta}$, and being symmetric with respect to the radius $\left\{r e^{i \theta}, 0 \leq r<1\right\}$. We assume that

$$
f\left(e^{i \theta}\right)=\lim f(z)
$$

holds uniformly in $e^{i \theta} \in K$ as $z \rightarrow e^{i \theta}$ inside $T(\theta, \alpha)$. Now fix $p \in(0, \infty)$. Since $f \in H^{p}(D)$, the radial limits $f\left(e^{i \theta}\right), 0 \leq \theta<2 \pi$, belong to $L^{p}(d \theta)$. We assume that $K$ is included in the Lebesgue set for $f$ and that

$$
\frac{1}{2 r} \int_{\theta-r}^{\theta+r}\left|f\left(e^{i \varphi}\right)-f\left(e^{i \theta}\right)\right|^{p} d \varphi \rightarrow 0
$$


uniformly in $e^{i \theta} \in K$ as $r \rightarrow 0$. Such a set $K$ can be found with $\left|\bar{F}_{n t} \backslash K\right|$ as small we please.

Fix $\delta>0$ so small that $\left|f\left(e^{i \theta}\right)-f(z)\right|<\epsilon$ if $e^{i \theta} \in K,|z|>1-\delta$ and $z \in T(\theta, \alpha)$. We are now in a convenient position for applying Vitushkin's scheme for approximation (see [13] or [4]). Let $\left\{\Delta_{j}\right\}_{j=1}^{N}$ be a finite collection of open discs with centers $z_{j} \in K$ and a common radius $r<\delta$. Following Vitushkin's scheme, let $\varphi_{j} \in C_{0}^{1}\left(\Delta_{j}\right)$ be chosen such that $0 \leq \varphi \leq 1$ and $\varphi \equiv 1$ in $\Delta_{j}^{1}=\left\{z:\left|z-z_{j}\right|<\frac{r}{2}\right\}$. As a preliminary approximation to $f$ we define

$$
f_{K}=f-G_{K}
$$

where $G_{K}=\sum_{j} T_{\varphi j}\left(f-f\left(z_{j}\right)\right)-r_{j}$ is a finite sum which we shall explain in some detail.

We assume $f$ is defined outside of $D$ by $f\left(\bar{z}^{-1}\right)=f(z)$. For general properties of the $T_{\varphi}$-operator we refer to [11] or [4, page 30]. Here we only note that

$$
\begin{aligned}
T_{\varphi j}\left(f-f\left(z_{j}\right)\right)(\varsigma) & =\varphi_{j}(\varsigma)\left(f(\varsigma)-f\left(z_{j}\right)\right) \\
& -\frac{1}{\pi} \int_{\Delta_{j}} \int \frac{f(z)-f\left(z_{j}\right)}{z-\varsigma} \frac{\partial \varphi_{j}}{\partial \bar{z}} d x d y(z) \\
& =U_{j}+V_{j} \text { say. }
\end{aligned}
$$

We assume $\left|\frac{\partial \varphi_{j}}{\partial \bar{z}}\right| \leq \frac{A}{r}$, where $A$ is a numerical constant. Since $f \in$ $H^{p}(D)$, we have in particular that $f \in L^{p}(d x d y)$ locally. Therefore the convolution term $V_{j}$ is continuous as a function of $\varsigma$. If $\alpha$ is close to $\pi$, Hölders inequality gives that

$$
\left|V_{j}(\varsigma)\right| \leq \epsilon, \quad \varsigma \in \mathcal{C} \quad j=1,2 \ldots N .
$$

Note also that $V_{j}$ is analytic outside $\Delta_{j}$. According to Vitushkin's scheme, the functions $r_{j}$ should be analytic outside a compact subset of $\Delta_{j} \backslash \bar{D}$ and with the property that $\left(V_{j}-r_{j}\right)(\varsigma)$ has a zero of order 3 at $\infty$. In addition we should require

$$
\left\|r_{j}\right\|_{\infty} \leq A_{1}\left\|V_{j}\right\|_{\infty} \leq A_{1} \epsilon, \quad j=1,2, \ldots, N
$$

where $A_{1}$ is a numerical constant. In our simple situation, the existence of $\left\{r_{j}\right\}$ is rather evident ([4, page 210-214]). From the individual bounds $(3)$, it is part of Vitushkin's scheme that

$$
\left\|\sum_{j=1}^{N}\left(V_{j}-r_{j}\right)\right\|_{\infty} \leq A_{2} \epsilon
$$


for some numerical constant $A_{2}$. We have not claimed that $\left\{\Delta_{j}^{1}\right\}_{j=1}^{N}$ cover all of $K$. In fact we shall assume that $\Delta_{j} \cap \Delta_{k}=\phi$ if $j \neq k$. In addition we assume that

$$
\left|K \cap \bigcup_{1}^{N} \Delta_{j}^{1}\right| \geq A_{3}|K|
$$

for some numerical constant $A_{3}$, where $\Delta_{j}^{1}=\left\{z:\left|z-z_{j}\right| \leq \frac{r}{2}\right\}$. We remark that $f_{K}=f-G_{K}$ is analytic near $\Delta_{j}^{1} \cap T$ for $1 \leq j \leq N$. This is seen by writing

$$
f_{k}=\left(f-U_{j}\right)-V_{j}-\sum_{i \neq j}\left(U_{i}+V_{i}\right)+\sum_{i=1}^{n} r_{i}
$$

and inspecting these four terms separately.

Note that the (Fatou) boundary values $f\left(z_{j}\right)$ satisfy $\left|f\left(z_{j}\right)\right| \leq\|f\|_{F}$. Since

$$
f_{K}=f\left(1-\sum \varphi_{j}\right)+\sum_{j} \varphi_{j} f\left(z_{j}\right)+\sum_{j}\left(V_{j}-r_{j}\right)
$$

we have

$$
\left\|f_{K}\right\|_{F} \leq\|f\|_{F}+A_{2} \epsilon .
$$

From (3) and (5) we also get

$$
\left\|f-f_{K}\right\|_{H^{p}(D)}=\left\|G_{K}\right\|_{H^{p}(D)} \leq\left(1+A_{2}\right) \epsilon
$$

if $r$ is sufficiently small.

The function $f_{K}$ satisfies the conditions for $f_{1}$ in Lemma 1 except that $f_{K}$ is only analytic (and hence continuous) near a subset $P_{K}$ of $K$. But since $\left|P_{K}\right| \geq A_{3}|K|$, Lemma 1 follows by repeating our construction countably many times. The main reason why repetition works, is that the $T \varphi$-operator preserves continuity and analyticity ([4, page 30$])$.

It remains to show that $C_{u a}(\hat{F}) \subset \overline{\left.H^{p}(D)\right|_{\hat{F}}}$. Let $B$ denote the Banach algebra $\overline{\left.H^{\infty}(D)\right|_{\hat{F}}}$. Also put $X=(\hat{F})$. If $V$ is a component of $\mathcal{C} \backslash X$, there mus exist $h \in H^{\infty}(D)$ such that

$$
1=h\left(z_{0}\right)>\|h\|_{F}
$$

for some $z_{0} \in V$. But then $1-h$ is invertible in $B$ and since

$$
1-h=\left(z-z_{0}\right) g, \quad g \in H^{\infty}(D)
$$


we conclude that $\left.\left(z-z_{0}\right)^{-1}\right|_{\hat{F}} \in B$. This means that $\left.R(X)\right|_{\hat{F}} \subset B$, where $R(X)$ is the uniform closure on $X$ by the rational functions with poles off $X$.

But if $\left\{V_{j}\right\}$ are the components os $\mathcal{C} \backslash X$, the maximum principle gives $\partial V_{j} \cap T \neq \phi, j=1,2, \ldots$ and hence $\partial X=\cup_{1}^{\infty} \partial V_{j}$. For such sets $X$ (with empty "inner boundary") Vitushkin has proved that $R(X)=C_{u a}(X)$ ([4, page 219]), and hence Theorem 1 is proved.

This solves completely problem 8.5 no. 2 in $[7]$ for the space $H^{p}(D)$, $0<p<\infty$. For $p=\infty$ the problem is still open.

For $p=\infty$, some information about $\overline{\left.H^{\infty}\right|_{F}}$ can be obtained from the work by Carl Sundberg in [12]. If $f \in \mathrm{BMOA}$ and $\left.f\right|_{F}$ is bounded, Sundberg shows that $f \in \overline{\left.H^{\infty}\right|_{F}}$. On the other hand, our proof above shows that any $f \in \overline{\left.H^{\infty}\right|_{F}}$ can be written as $f=u+v$ with $u \in H^{\infty}$ and $v \in \cap_{p>0} H^{p}(D)$. Several questions arises from this. Here we only mention the following: Let $f \in$ BMOA be bounded on a relatively closed set $F \subset D$.

Is there $g \in H^{\infty}$ such that the restriction $\left.(f-g)\right|_{F}$ is uniformly continuous on $F$ ?

\section{References}

1. A. M. DAvie AND A. Stray, Interpolation sets for analytic functions, Pacific J. Math. 42(1) (1972).

2. J. Detraz, Algebres de fonctions analytiques dans le disque, Ann. Sci. Ecole Norm. Sup. 4e serie (1970), 313-352.

3. P. L. Duren, "Theory of $H^{p}$ spaces," Academic Press, 1970.

4. T. W. Gamelin, "Uniform Algebras," Prentice Hall, Englewood Cliffs, N. J., 1969.

5. J. Garnett, "Bounded Analytic Functions," Academic Press, 1980.

6. S. N. Mergelyan, Uniform approximation to functions of a complex variable, Urephi Mat. Nauk. 7(2) (1952), 31-122.

7. ?, "Linear and Complex Analysis Problem Book," Lecture Notes in Mathematics 1043, Springer Verlag, 1984.

8. F. Perez-Gonzalez And A. Stray, Farrell and Mergelyan sets for $H^{p}$-spaces, $0<p<1$, Michigan Math. J. 36 (1989), 379-386.

9. A. Stray, Decomposition of approximable functions, Ann. of Math. 120 (1984), 225-235.

10. A. Stray, Approximation by analytic functions which are uniformly continuous on a subset of their domain of definition, American J. Math. 99 (1977), 787-800. 
11. A. Stray, Characterization of Mergelyan sets, Proc. Amer. Math. Soc. 44 (1974), 347-352.

12. C. SundBerg, Truncations of BMO functions, Indiana University Math. I. 33(5) (1984), 749-779.

13. A. G. Vitushrin, The analytic capacity of sets and problems in approximation theory, Russian Math. Surveys 22 (1967), 139-200.

\author{
Department of Mathematics \\ University of Bergen \\ Allégt 55 \\ 5007 Bergen \\ NORWAY
}

Primera versió rebuda el 2 de Setembre de 1993, darrera versió rebuda el 9 de Febrer de 1995 